\title{
Data Sharing between Local and National Governments for the Benefit of the Citizen
}

\author{
Online Free School Meals as a Transformational Project
}

\author{
Alan Strickley \\ CRIA Technologies UK \\ alan.strickley@criatech.co.uk
}

\begin{abstract}
The Online Free School Meals (OFSM) project [1] is an example of transformation through information technology (IT) in local and central government. It brings together several central government departments in England, removes a bureaucratic process for the citizen, speeds up the process significantly, saves money, removes the stigma of benefit claim, and enables automatic audit and renewal [2]. The OFSM project was led by Connect Digitally, the team that introduced online school admissions across England [3]. The project brief was to develop a solution which would check parent/carer eligibility based on minimal criteria across the three central government databases which held the essential data, namely, Her Majesty's Revenues and Customs (HMRC), the Home Office (HO) and the Department for Work and Pension (DWP). The project resulted in the Eligibility Checking Service (ECS, previously called the Hub) [4] which from three key data items - family name, date of birth, and National Insurance number (NINO) or National Asylum Support Service (NAAS) registration number - can deliver an immediate eligibility response in over $90 \%$ [5] of applications via a web interface. The large response from local authorities (LAs) across England and Wales led to over 149 using at least some of the facilities of the ECS [6] and many incorporating the full web service process, saving money, time and delivering a beneficial service for the citizen. This paper looks at the original process, the development of the system, the current take-up and the future of OFSM in England.
\end{abstract}

Keywords: Online free schools means, eligibility checking service, Connect Digitally, Department for Education.

\section{The Free School Meals Application for Eligibility Process}

Access to free school meals (FSM) in England and Wales is a means-tested benefit dependent upon the circumstances of the parent/carer [7]. Eligibility for this benefit enables the children of the parent/carer to receive free school meals and free milk at the school they attend. As a key indicator of deprivation, FSM eligibility is currently used for other benefits such as Pupil Premium [8] and Early Years' funding [9]. As such, it is becoming a key indicator and funding conduit for Local Authorities (LAs) 
and schools. Originally, the process of applying for the benefit was bureaucratic, time consuming and stigmatised for the applicant, requiring paper application and proof of benefit to be presented to the school or/and the LA on an annual basis. From 6 April 2010 these criteria applied to children whose parents were in receipt of the following support payments [4]: Income Support (IS); Income Based Job Seeker's Allowance (IBJSA); Support under part VI of the Immigration and Asylum Act 1999; Child Tax Credit, provided that their annual income was less than $£ 16,190$ (as assessed by HMRC) and they were not in receipt of Working Tax Credit; The Guarantee element of State Pension Credit; and Income Related Employment Support Allowance.

In addition, children who received Income Support or Income Based Job Seeker's Allowance in their own right were also entitled to receive free school meals. It should also be noted that children who received "education suitable for children who have not attained compulsory school age" would need to satisfy the additional requirement of "receiving education both before and after the lunch period", before being eligible to receive free school meals.

The FSM service is administered by LAs, although in some cases this function is devolved to schools within that LA. Until 2009 the application was generally a paper-based process in which the parent/carer was also required to submit proof of eligibility to the LA in which they lived or to the school their children were attending. Examples of evidence might be a letter from the Job Centre, a payment book, a pension book, asylum seekers documentation, etc. Generally the LA or other checking authority would take the documentation supplied by the parent on face value, although sometimes further manual checks with the appropriate authority were required. The process for the parent of supplying these documents could be time consuming and the checking could take several weeks if done manually with the appropriate authorities.

For automated electronic checking, the data that would enable LAs to check the eligibility criteria directly are held by 3 central government agencies: DWP; HMRC; and HO.

\section{Parental Experience in Applying for FSM Manually}

Applying for FSM using a manual method can be lengthy and can involve a large amount of form filling, visits and postage. Figure 1 [10] shows the 5 steps of the process.

Awareness. In order to apply for FSM the parent will need to be aware that the benefit is available. Agencies that give support may not be aware of or inform the parent of FSM benefits. This can lead to an extended period in which the child does not receive a proper meal or the parent suffers financially.

Proving. When the parent is aware of the benefit, they will need to obtain the appropriate documentary evidence from the supporting agency (examples as described above). This may require a visit, letter or other method and may take several weeks. 
Applying. Once the evidence has been obtained, the parent may complete the application. The form will then need to be delivered or taken to the school or LA.

Receiving. The appropriate authority will look at the evidence and make a decision. This may take several days or weeks in more complex cases. Eventually the child will receive the free meal entitlement and if appropriate back payment may be made although this can take several weeks.

Reapplying. After a year there is a requirement to reapply, although notification may not be automatic in which case the child may miss out on the entitlement. The process will need to recommence from step 1 .

This, in addition to the stigma attached to applying for the evidence to support the FSM status, often acts as a disincentive to parent/carers to continue their application or even start it in the first place. This can have detrimental effects on their children's health and attainment [2].

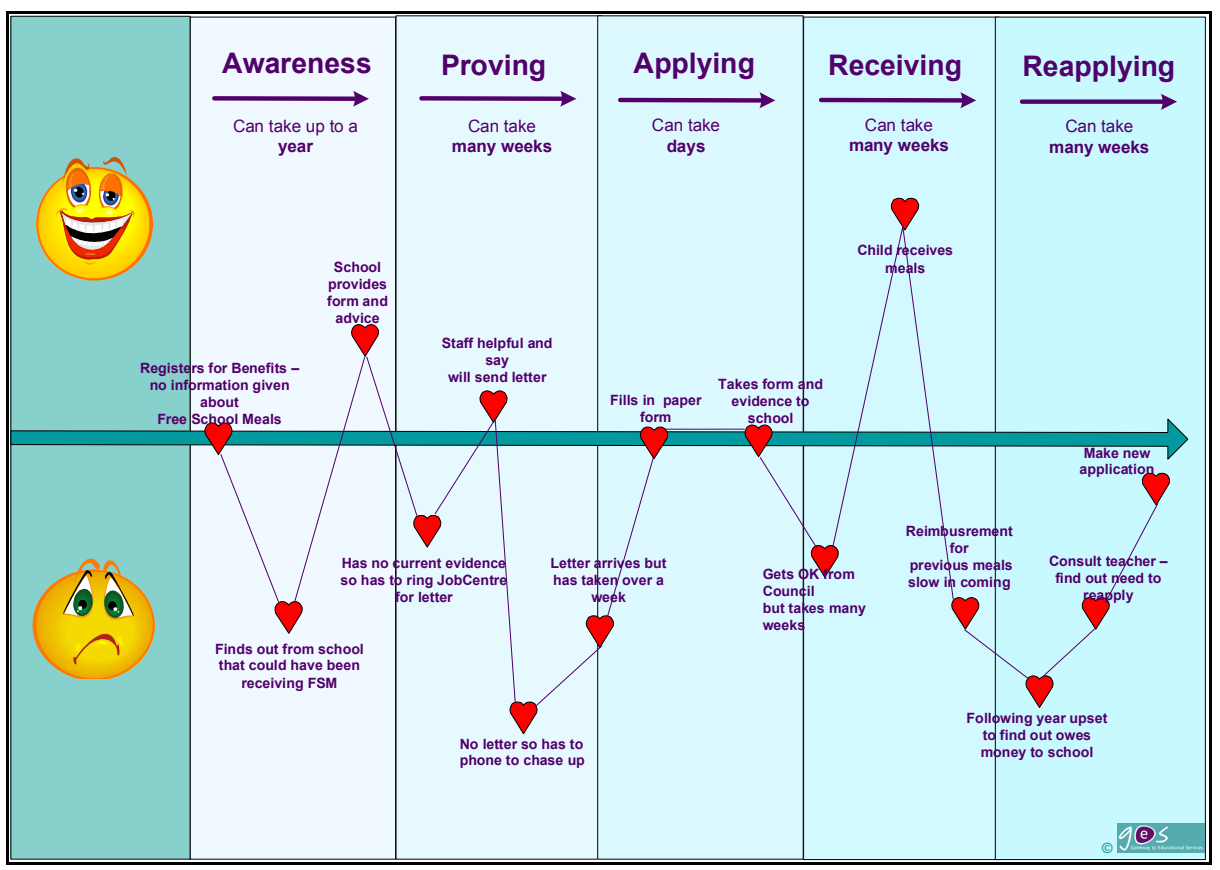

Fig. 1. Customer Journey before OFSM

Figure 1 shows the highs and lows of applying for FSM before the OFSM project was completed [10]. As can be seen, there are considerably more lows than highs and the time line can be extensive. 


\section{The OFSM Project}

In 2007, in line with the Varney Review [1], which highlighted the need for social inclusion, joined up government and citizen empowerment (citizen centric services), a project was initiated to cover the following recommendations of this review:

- Promoting social inclusion.

- Demonstrating data sharing between central government agencies and LAs enabling LAs to check eligibility criteria for FSM without requiring paper documentation from the parent/carer.

- Illustrating joined up government in action: central and local government working together for the benefit of the citizen.

- Designing a service around the citizen: removing the burden of proof from the parent/carer and eliminating a potential barrier where parents/carers feel stigma if they have a face to face meeting regarding FSM.

- Transforming the delivery of a national service namely FSM.

As a project, OFSM highlighted a number of issues and challenges. These were:

- Data sharing between central and local government.

- Legal gateways.

- Differences between different types of LAs.

- Equality of services provided to citizens served by 150 LAs.

The eAdmissions National Project [3], led by Hertfordshire County Council, had 3 years' experience in working with the 150 LAs on the delivery of an online school admissions service for parents/carers. The project's success in redesigning the service from the parents'/carers' perspective was demonstrated by an $18 \%$ national take-up of the online service in the first year: an unprecedented level of take-up for a national service provided by LAs [3]. As FSM fell within the Department for Children Schools and Families (DCSF) (now the Department for Education, DfE) policy area and DCSF were keen to use eAdmissions' expertise and knowledge to inform the online FSM project, it was agreed that DCSF and members of the eAdmissions National Project team would work with the Cabinet Office and Tameside LA on the development of online FSM, within the wider DCSF programme the "Gateway to Educational Services" (GES, this later became known as Connect Digitally and the names will be used to be historically accurate but are synonymous).

The project was to look at the feasibility of linking the three major central departmental databases that contained the information that could be used to evidence a parent/carer's eligibility to FSM for their children.

Clearly this was to be a complex project requiring agreement by all stakeholders, cooperation across central and local government, user acceptance and security. 


\section{The Issues}

One issue was that unitary/metropolitan councils are responsible for a different set of citizen services from county councils, although FSM is a service provided by all of the 150 English LAs (now 152) responsible for education. In addition, unitary/metropolitan LAs administer housing benefit and council tax benefit and in this role have access to the Citizen Information Service (CIS) provided by DWP. The CIS system identifies if a citizen (parent/carer) has been awarded income support or job seeker's allowance: eligibility criteria for FSM. As a result, a proposal was made that the CIS system could be used by LAs to determine eligibility criteria for FSM. This would enable the majority of FSM applications to be resolved, though not for applications requiring access to HMRC or HO data. However, DWP and DCSF lawyers raised doubts as to the legality of this approach and in addition LAs do not have access to CIS, and to achieve true transformation, equality of service had to be provided to all citizens. The LAs are responsible for administering around 30\% of FSM resulting in a large proportion of the country being excluded from the project.

It was agreed that to achieve genuine transformation, data sharing must be enabled for all LAs providing FSM and as such the use of the CIS was dropped.

\section{The Solution}

The solution was the creation of the DCSF FSM hub, a unique system designed to streamline the process for FSM eligibility checking and reduce the burden on parents/carers and LAs. The system allows LA users to undertake record checks from one central point, the DCSF Hub (now the Eligibility Checking Service, ECS), of the data held by the DWP, HMRC and HO to establish FSM eligibility. Checks may be performed for an individual application or in batch mode.

As a result parents/carers no longer need to provide paper evidence of the relevant tax or benefit information to confirm FSM eligibility. A central check on National Insurance number (NINO) or National Asylum Support Service (NASS) reference number, family name and date of birth are sufficient to confirm eligibility. Basically the ECS acts as an interface to the three central government databases. These are HMRC, for data on tax credits, DWP, for data on income and benefits, and HO, for data on asylum seekers. A web interface enables the LA to check against the applicants three key data items (family name, NINO or NAAS and date of birth) and a response from the ECS is almost immediate, resulting in a 'Found' (Eligible) or 'Not Found' (not known) response. A 'Not Found' response will indicate that the applicant is not eligible or that their data is missing for some reason. Because of a delay in updating some of the central databases and some missing data an eligible applicant may be flagged as 'Not Found' when they are in fact genuinely eligible. This can be resolved by resubmitting the application the next day or week or by the parent presenting paper evidence to the LA or school. Despite this slight disadvantage of the 
system, it has been shown to identify over $90 \%$ of queries correctly [6]. The system allows details to be checked as a one-off or in a batch of applications. It can also be used to check renewals on a regular basis as well as audits of existing eligibility.

\section{The OFSM System as an Incremental Citizen Facility}

OFSM using the ECS may be developed by LAs through a variety of increments, each giving more value and effectiveness.

1. Use the DfE ECS for FSM applications, renewals and audit. Whilst the application may still be made through non-IT methods, e.g. phone, visit; paper form, there is no need for the LA to check the accompanying paper evidence as the LA is able to check eligibility through the use of the ECS using single or batch process. The use of the ECS for audit of existing eligibilities on a periodic basis removes the need for re-application by the parent and will indicate where eligibility has lapsed.

2. Improve the LA business process and remove the need for paper proof of benefit at application and renewal. The removal of the requirement for the parent to supply eligibility evidence on application removes much of the storage and retrieval issues for the LA and makes the process easier for the parent.

3. Introduce online forms for parents/carers to apply for FSM. The introduction of an online form means that the parent no longer has to use paper-based or personal methods to communicate their application for FSM.

4. Use web services to provide a seamless OFSM service to parents/carers. Web services ensure that the parent and LA gets a response in real time to their applications.

5. Work with schools, suppliers and caterers to ensure that free school meals are provided for eligible children by the start of the next working week. In tandem with stage 4, the use of electronic methods to communicate eligibility to schools ensures that the meal can be provided as soon as possible, ideally the next day.

\section{$7 \quad$ A Transformed FSM Service}

The successful rollout of the DfE ECS has enabled LAs to provide a more streamlined service. The burden of proof has been removed from parents/carers eliminating the paper documents currently required by LAs. The timescale for responding to a FSM application has be reduced to between 24 to 48 hours instead of, in some cases, several weeks.

Building on the experiences gained with the proof of concept models in Tameside and Hertfordshire LAs, the long-term goal was to provide the solution outlined for all LAs: a secure online service to parents/carers, linked via a web service to the Hub, enabling an immediate response to the application and direct notification to the appropriate school. At the time of writing, there are 17 LAs utilising the full service. 


\section{Parental Experiences with OFSM}

Figure 1 illustrated the parental experience of applying for FSM using the old paper method. Figure 2 [10] shows the experience using a full web enabled OFSM system. As can be seen, the whole process is faster, streamlined and avoids stigma.

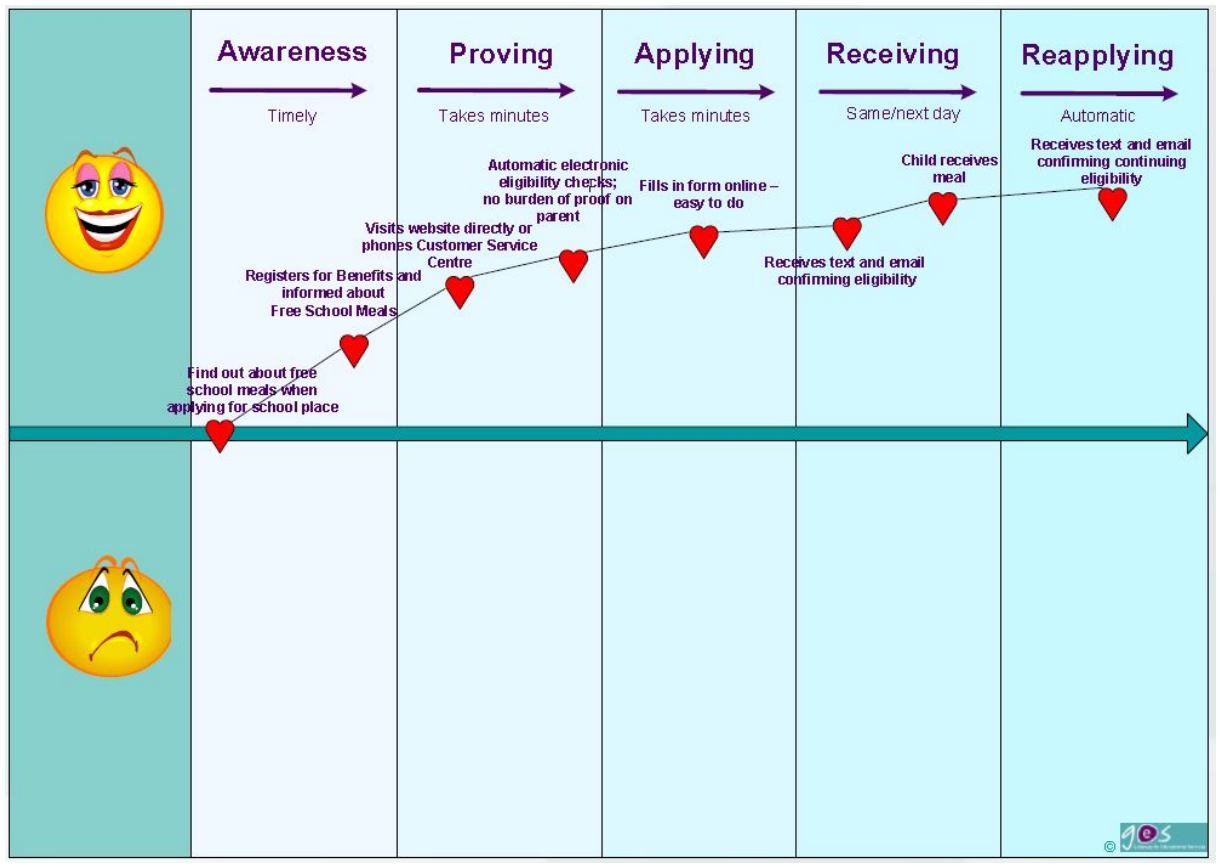

Fig. 2. Customer journey with OFSM

It also has efficiency and financial advantages for the LAs, discussed later in this paper.

\section{Uptake of ECS Usage}

The take-up in usage of the ECS has been high. Figure 3 [5] shows the number of LAs using the ECS between 2008 and September 2011, on a month-by-month basis. As can be seen, the number of LAs has increased month on month across the 3 year period. This will level out as we reach saturation point, i.e. all LAs using the system. These data show all types of usage from one-off checks to full web serviced systems. As the system becomes more embedded, LAs will move through stages of maturity. 


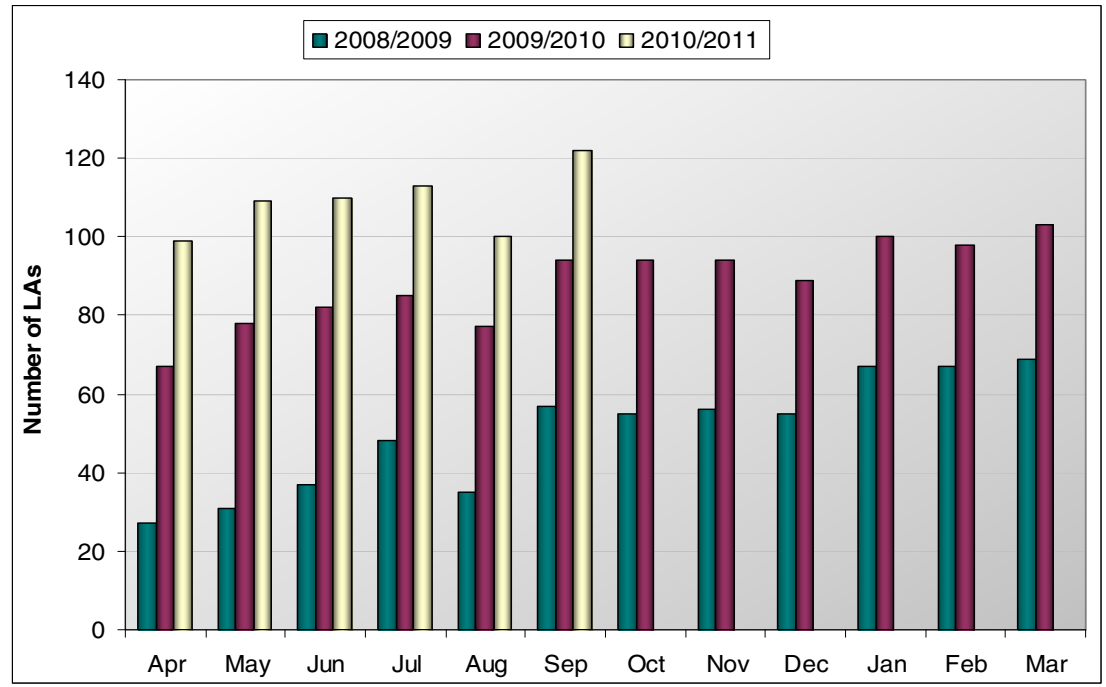

Fig. 3. Number of English LAs using the FSM ECS

Figure 4 [5] shows the number of queries made to the ECS on a month on month basis across the same 3 year period. It can be seen that the number of queries is not only increasing with the number of LAs using it, but also as a result of the use of the ECS for audit purposes as well as normal applications. Some LAs audit as frequently as weekly, meaning that as soon as an applicant becomes ineligible the FSM can be stopped. Alternatively where an applicant has been indicated as ineligible, the audit may find they are now eligible and the FSM may be resumed.

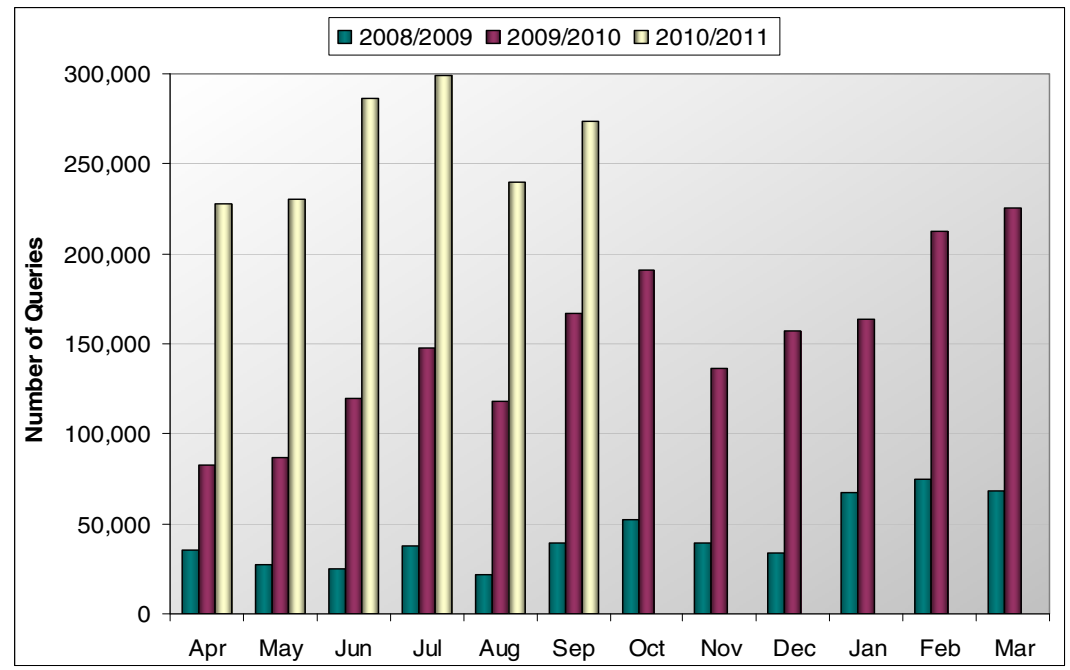

Fig. 4. Number of FSM ECS queries by English LAs 
Figure 5 [5] shows the number of ECS queries each month in 2009 and 2010 as batch and online. It is interesting to note that although the number of batch queries has increased, the number of online ones has increased less so. This results from the considerable extra work involved in setting up an online system with less financial benefits. As a result, only 17 LAs currently have this feature, but these do represent a large number of queries showing that the process is well used.

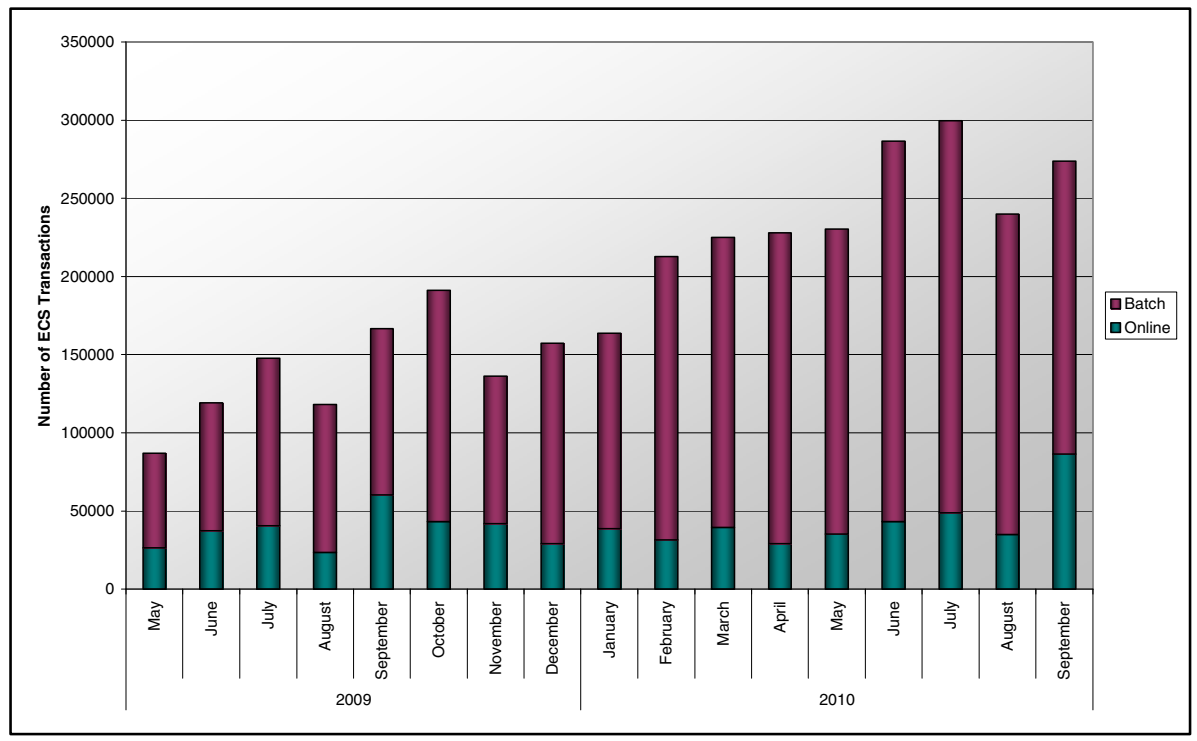

Fig. 5. Number of ECS queries each month (England).

\section{Current Position}

The latest figures show take-up of the ECS across England and Wales [5]. Of the 152 local authorities (LAs) in England with responsibility for education, 143 (94\%) used the DfE FSM ECS in November 2011. One hundred and fifty (98\%) LAs used the ECS since the service started.

There were 527,569 transactions within England and Wales during November 2011: 430,544 were batch queries; and 97,025 were single queries. Currently 39 LAs run audits at least once a month and 29 more LAs run audits at least once a term. One hundred and fifteen English LAs no longer ask for paper proof of benefit to process free school meal claims.

In addition to providing a better system for the citizen, the use of the ECS has resulted in efficiencies, demonstrated below by evidence from various LAs across England and Wales [5]:

- 10,000 fewer citizen call, saving $£ 20,000$.

- Reduction in staff saves $£ 98,000$, and reduction in office space saves $£ 6,000$.

- Fewer queries from schools saves $£ 5,000$, and improved audit process $£ 18,000$. 
- Expenditure on leaflets, postage and manual entry of applications, saves $£ 63,000$.

- Booklet printing not needed, saves $£ 49,000$.

- Data input by temporary staff reduced from 42 weeks to 5 weeks, saving $£ 17,000$.

- Since October 2009, Hertfordshire LA identified approximately 4,000 recipients no longer eligible for FSM. It was estimated that, through not providing a meal to these recipients, around $£ 100,000$ was saved for 2010 to 2011 .

\section{An Example of the OFSM Web Service in Practice}

A typical system using full web services would consist of the following elements:

Online Form. An online form needs to be available for the applicant from the LA website. This may be hosted within the LA's own infrastructure or rented from a cloud provider. The form will need to comply with the appropriate standards of the LA and usability guidelines. The form will need to capture applicant family name, NINO or NAAN and date of birth as a minimum; it is likely that address and contact information together with pupil data will also be required.

LA Back Office System. The results of the ECS check will be relayed to the parent, the school and the LA. There will need to be a back office system capable of holding this eligibility information including the data captured on the form. This will be required for renewal, audits and queries from the applicants. For the parent, results will be instantly available on screen and additionally by email or SMS as appropriate.

School Back Office System. Ideally the school back office will offer a narrower view of the LA system for that school only. Where eligibility checking is administered by the school itself they will be able to make changes which in turn will be reflected in the LA's view.

Data Transfer to Schools. Although the information regarding eligibility may be transferred to the school via email, similar transfer to the school might be in a format imported directly into their management information system (MIS) via a common transfer file (CTF), system interoperability framework (SIF) or similar.

\section{The Success of the Project}

The project has been a success, evidenced by the following awards:

- Shortlisted for the e-Government National Awards 2010.

- Local e-Government Excellence -Take-up and Usage Growth, November 2010.

- Winner Civil Service Awards - Public Service Award, November 2010.

- Runner-up for the Civil Service Communication Award, November 2010.

- Medallist, BCS UK IT Industry Awards - Public Sector Project of the Year, Project Excellence Section, November 2010. 
- Winner Local Government IT Excellence Award, Service Transformation Category.

- Winner Good Communications IT Partnership Award, June 2010.

- Winner Overall Government Computing Award, April 2010.

- Winner Collaboration Government Computing Award, April 2010.

\section{The Future}

Since the development of the ECS, the use of FSM eligibility has increased as a key indicator of deprivation and as a conduit for funding, in particular, the introduction of the Pupil Premium which is an additional payment, on a per pupil basis, made directly to school for children from poor backgrounds. This premium is currently based on FSM eligibility and is soon to become based on any FSM eligibility in the last 6 years [8]. This will be an incentive for schools to encourage parents to apply for the benefit even if they do not wish to take the free meals.

In the future there is likely to be an increase in the number of parent/carers who go through the process of eligibility checks for FSM as a result of school pressure for Pupil Premium payments, lobbying from the School Food Trust (for healthier lunches), changes to the admissions criteria (allowing admissions priority based on FSM eligibility), and general austerity effects nationally.

The system described here will make it easier and more effective and efficient for both parent/carer and administration body, be that school or LA. In addition it should, in the longer term, make the actual FSM eligibility numbers that are used as a measure of deprivation for a large number of statistical measures a better reflection of the true position nationally. In addition, Early Years' funding is likely to be driven by FSM eligibility in the near future [9], again making use of a simple eligibility checking system an essential LA tool.

The increase in the number of Academies and Free Schools (state schools that are independent from LA control) will also have an impact on, in particular, the use of web services. This is because the legal framework for the ECS only allows LAs to have access. Academies may use their own LA but this may not be viable (the LA may charge or simple refuse to assist). As independent bodies, the academies may use any LA system through negotiation. Alternatively, a virtual LA may be set up for this particular purpose. This solution would require a full and seamless system using web services, probably using a cloud infrastructure as discussed below. The OFSM data capture form is likely to be similar regardless of which LA (or academy) is using it. The only real difference might be the branding with the school or LA banner. The use of cloud services for the form which incorporates web services for communication with the ECS would seem a sensible and effective solution, particularly for small LAs, those with fewer FSM pupils, and academies. Such a project is underway in conjunction with Software for Data Analysis [12] and Hampshire County Council. 


\section{Conclusions}

The success of the project depended on two main criteria: an effective approach to collaboration and stakeholder engagement [13]; and a transformation that saved time, money, removed stigma and better served the citizen. There are other development areas being considered, particularly those which require paper proof of eligibility through local and national data sets, for example, school transport and free bus passes.

\section{References}

1. Connect Digitally: The OFSM Project (2012), accessible at http: / /www. communities.idea.gov.uk/c/1875909/forum/ thread. do? backlink $=$ ref \&id $=4489865$ \& themeId $=3008216$

2. School Food Trust: Home page (2012), accessible at http://www.schoolfoodtrust.org.uk/

3. Connect Digitally: The Online School Admissions Project (2012), accessible at http://www.communities.idea.gov.uk/c/1875909/forum/theme-index.do?themeId=3008219

4. DfE: The Eligibility Checking Service (2012), accessible at http: / /www. communities.idea.gov.uk/c/1875909/doclib/ document-display.do?backlink=ref\&id=8356480\&themeId=3008216

5. Connect Digitally: OFSM statistics (2012), accessible at http://www.communities.idea.gov.uk/c/1875909/doclib/ index. do?pagesize=999

6. Connect Digitally: Latest figures for ECS usage (2012), accessible at http://www. communities.idea.gov.uk/c/1875909/forum/ thread. do?backlink $=$ ref\&id $=12048567 \&$ themeId $=3008216$

7. HMSO: The Education Act 1996 As amended by the School Standards and Framework Act 1998, the Education Act 2002, the Anti-Social Behaviour Act 2003, the Education Act 1996 (Electronic Communications) Order 2004 (SI2004/2521), the Education Act 2005, the Childcare Act 2006, the Education and Inspections Act 2006, and the Welfare Reform Act 2007. TSO, London (1996)

8. DfE: Pupil Premium: What you need to know (2012), accessible at http://www.education.gov.uk/schools/pupilsupport/premium/ a0076063/pupil-premium-what-you-need-to-know

9. DfE: Supporting Families in the Foundation Years: Proposed Changes to the Entitlement to Free Early Education and Childcare Sufficiency (2012), accessible at http://www. education.gov.uk/consultations/index. cfm?action= consultationDetails\&consultationId=1782\&external=no\&menu=1

10. Connect Digitally: OFSM Customer Journeys (2012), accessible at http: / / www. communities.idea.gov.uk/c/1875909/doclib/ document-display.do?backlink=ref\&id=490255

11. Varney. D.: The Varney Review (2012), accessible at: http: / /www. oakleigh.co.uk/page/1568/News/News-Archive/ Articles/The-Varney-Review-an-analysis

12. SDA: Home page (2012), accessible at https://www.cloudforedu.org.uk/ofsm/

13. Strickley, A., Spiers, T.: Successful implementation and increase of citizen take-up of egovernment: processes, strategies, practice and lessons learned. In: European Conference on e-Government 2012 Proceedings. ACI, Reading (2012) 